\title{
Anesthetic management of non-cardiac surgery with adult onset type of cor triatriatum sinister -A case report-
}

\author{
Hae Mi Lee, Hyun Suk Sung, and Sae Yeon Kim \\ Department of Anesthesiology and Pain Medicine, School of Medicine, Yeungnam University, Daegu, Korea
}

A 45-year-old woman with cor triatriatum sinister was admitted for laparoscopic resection of an ovarian tumor. Her medical history was benign with the exception of a single episode of syncope one year ago. A 1.5-cm membrane fenestration was found on echocardiography, but there were no other cardiac structural anomalies. General anesthesia was established with etomidate, sevoflurane, and remifentanil; no notable events occurred during the anesthesia. As cor triatriatum shows a clinical picture of mitral stenosis (MS), careful anesthetic management is required. (Korean J Anesthesiol 2011; 60: 444-448)

Key Words: Congenital heart disease, Cor triatriatum, General anesthesia.

Cor triatriatum sinister, first described by Church in 1868, is a rare congenital cardiac malformation in which a fibromuscular membrane divides the left atrium into two chambers [1]. Cor triatriatum sinister can occur as an isolated anomaly in approximately $30 \%$ of cases; however, cor triatriatum sinister is frequently associated with other cardiac anomalies, such as patent ductus arteriosus, atrial septal defects, anomalous pulmonary venous drainage, and left superior vena cava $[1,2]$. Although symptoms usually present in infancy, cor triatriatum sinister can first occur in adulthood when the membrane contains large fenestration or other escape drainage exists [3]. As cor triatriatum sinister resembles the physiology of mitral stenosis (MS), careful preoperative evaluation and anesthetic management are required. We present a patient with cor tri- atriatum sinister who had undergone laparoscopic surgery uneventfully and discuss the anesthetic management.

\section{Case Report}

A 45-year-old G3P2 (57 kg and $156 \mathrm{~cm}$ ) was admitted for ovarian tumor resection. She had dull pain on the left lower abdomen for 3 months and a CT scan obtained at an outlying hospital suggested mature cystic teratomas on both ovaries.

The family history was non-contributory and the medical history was benign, including the obstetric history, with the exception of a single episode of syncope about 1 year ago. Headaches, palpitations, and chest discomfort began after the syncopal episode, therefore a brain MRI, ECG, and 24-hour

Received: November 22, 2010. Revised: December 21, 2010. Accepted: December 29, 2010.

Corresponding author: Sae Yeon Kim, M.D., Department of Anesthesiology and Pain Medicine, School of Medicine, Yeungnam University, Daemyung 5-dong, Nam-gu, Daegu 705-717, Korea. Tel: 82-53-620-3366, Fax: 82-53-626-5275, E-mail: sykim@med.yu.ac.kr

(c) This is an open-access article distributed under the terms of the Creative Commons Attribution Non-Commercial License (http:// creativecommons.org/licenses/by-nc/3.0/), which permits unrestricted non-commercial use, distribution, and reproduction in any medium, provided the original work is properly cited. 
holter ECG were obtained, all of which showed normal findings. However, trans-thoracic echocardiography (TTE) revealed mild pulmonary hypertension (RV end-systolic pressure, 35 mmHg; RA pressure, $5 \mathrm{mmHg}$; PCWP, $8.76 \mathrm{mmHg}$ ), a slightly thickened mitral valve (MV) with mild mitral regurgitation (MR), and an enlarged LA with a clear membrane across the LA. The systolic function of the left ventricle was normal (LVEF $=60 \%$ ) and there was no wall motion abnormality or visible thrombus (Fig. 1). For further details, a trans-esophageal echocardiography (TEE) and cardiac MRI were performed (Fig. 2 and 3A). A membranous structure divided the LA by two chambers and a $1.5-\mathrm{cm}$ fenestration was noted on the infero-medial side of the membrane. The antero-inferior chamber (distal chamber) contained a LA appendage and the orifice of the mitral valve, and the postero-superior chamber (proximal chamber) received blood from the pulmonary veins. Continuous wave Doppler tracing of the flow through the membrane showed a systolic wave reaching $0.97 \mathrm{~m} / \mathrm{s}$ and a diastolic wave reaching $1.23 \mathrm{~m} /$ $\mathrm{s}$. The mean pressure gradient across the membrane was 6.8 mmHg; no other cardiac anomalies were noted (Fig. 3B).

Under the diagnosis of cor triatriatum sinister, the patient was advised to undergo cardiac surgery first, but she declined. Because she had no other medical problems and symptoms suggestive of heart failure, a laparoscopic ovarian mass excision was planned. All pre-operative evaluations, such as ECG, chest $\mathrm{X}$ ray, and routine laboratory tests, were normal.

The pre-operative vital signs of this ASA class II patient were stable, as follows: blood pressure (BP), 140/90 $\mathrm{mmHg}$; heart rate (HR), 80 beats $/ \mathrm{min}$; and $\mathrm{SpO}_{2}, 99 \%$. Glycopyrrolate $(0.2 \mathrm{mg})$ was injected intramuscularly for pre-medication and an ECG (lead II), NIBP, $\mathrm{SpO}_{2}$, and bispectral index (BIS) were monitored during anesthesia. After 5 minutes of a remifentanil infusion $(0.20 \mu \mathrm{g} / \mathrm{kg} / \mathrm{min})$, anesthesia was administered with $10 \mathrm{mg}$ of

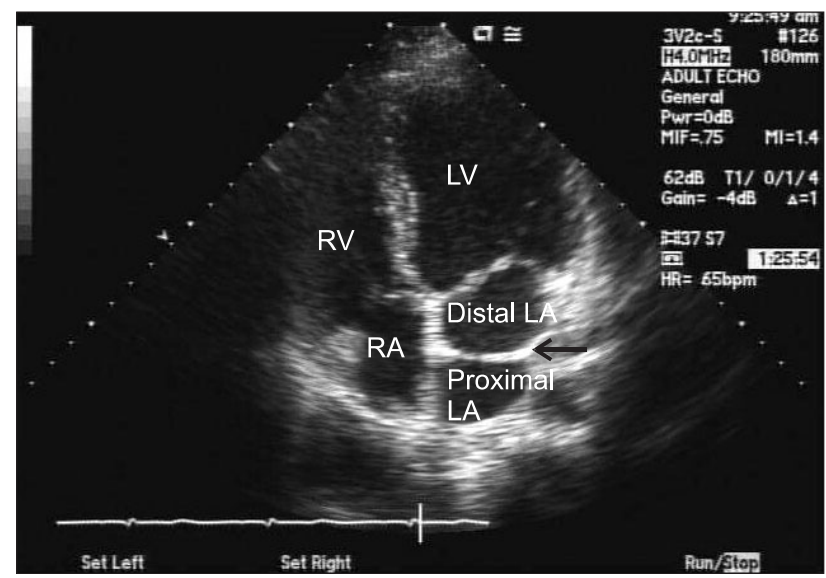

Fig. 1. Trans-thoracic echocardiogram in the four-chamber view, revealing the membrane within the left atrium (arrow). etomidate and $50 \mathrm{mg}$ of rocuronium bromide. Intubation was performed with a 7.5-mm inner diameter endotracheal tube and anesthesia was maintained with sevoflurane and an equal mixture of $\mathrm{O}_{2}$ and $\mathrm{N}_{2} \mathrm{O}$. The ventilator was set at a tidal volume $\left(\mathrm{V}_{\mathrm{T}}\right)$ of $500 \mathrm{ml}$ and a respiration rate $(\mathrm{RR})$ of 10 breaths $/ \mathrm{min}$. After preparation for surgery, $\mathrm{CO}_{2}$ gas was insufflated via a laparoscope port and a pneumoperitoneum was made with a pressure of $10 \mathrm{mmHg}$. While in the $30^{\circ}$ head-down position, the surgery was started and the ventilator setting was changed $\left(\mathrm{V}_{\mathrm{T}}\right.$ decreased to $450 \mathrm{ml}$; RR increased to $12-14$ breaths $/ \mathrm{min}$ ). The peak inspiratory pressure (PIP) increased from 17 to $20 \mathrm{mmHg}$ and the BP and HR increased slightly during the first 5 min after the $\mathrm{CO}_{2}$ pneumoperitonium was established. Remifentanil was titrated as follows during surgery; $\mathrm{BP}, 105 / 70-140 / 90 \mathrm{mmHg}$; $\mathrm{HR}, 65-90$ beats/min; $\mathrm{SpO}_{2}, 100 \%$; $\mathrm{EtCO}_{2}, 31-32 \mathrm{mmHg}$; and BIS, $42-48$. The surgery took $75 \mathrm{~min}$ without any notable events. Crystalloids $(600 \mathrm{ml})$ were infused and the total urine output was $40 \mathrm{ml}$. After reversal of the muscle relaxant, the patient was extubated and referred to a post-anesthesia care unit. During the 30-min recovery, the BP was 110/80-140/92, the $\mathrm{HR}$ was $80-95$ beats $/ \mathrm{min}$, and the $\mathrm{SpO}_{2}$ was $99 \%$ with $8 \mathrm{~L}$ of $\mathrm{O}_{2}$ via face mask. Post-operative pain was managed by a patientcontrolled analgesia (PCA) device with nalbuphine, and the patient was discharged after fully awakened from anesthesia. She was followed post-operatively in the Department of Thoracic Surgery.

\section{Discussion}

Cor triatriatum, also known as triatrial heart, is a rare con-

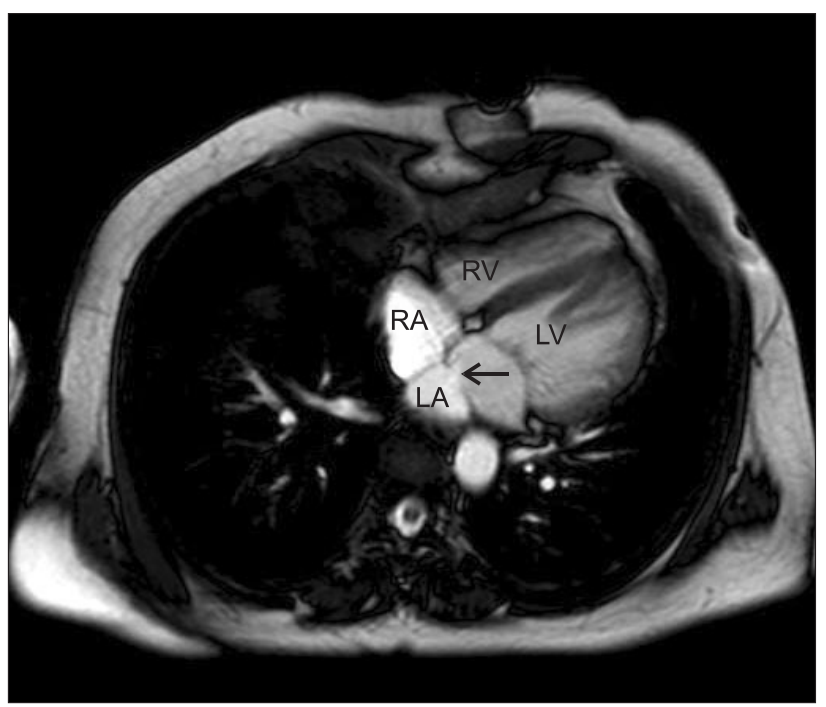

Fig. 2. MRI finding of cor triatriatum sinister. A membranous structure is dividing the left atrium by two chambers and a $1.5-\mathrm{cm}$ fenestration is noted in the middle of the membrane (arrow). 
genital cardiac malformation, the incidence of which has been reported from $0.1-0.4 \%$ of all congenital heart diseases [2] Many theories for cor triatriatum sinister have been suggested; however, the most widely accepted theory is that it results from a failure of incorporation of the common pulmonary vein into the left atrium during fetal life [4]. This abnormality involves subdivision of the LA by an oblique fibromuscular membrane that creates a proximal chamber receiving the pulmonary veins (accessory LA) and a distal chamber that includes the left atrial appendage and mitral valve (true LA) [5]. Most of these chambers are in communication with one or more openings in the membrane and depend upon the size and number of openings; the hemodynamics vary from normal to mimicking MS [1]. In most patients, the opening is severely restrictive and approximately $75 \%$ of those born with classical cor triatriatum (without an escape route) die in infancy [2]. However, the presentation can be delayed if there is a larger opening in the diaphragm and/or the LA communicates with the right atrium through an escape route, thus permitting partial decompression, such as a patent foramen ovale or atrial septal defect $[1,6]$.

Cor triatriatum produces symptoms by causing pulmonary venous obstruction and pressure overloading on the right side of the heart [6]. The most frequent initial symptoms in infants are respiratory distress, cyanosis, recurrent respiratory tract infections, and feeding difficulties, while older patients present with syncope, dyspnea, and hemoptysis. [2,6]. The LA progressively dilates due to pressure and volume overload, resulting in mitral regurgitation (MR), atrial fibrillation (AF), and right heart failure (RHF). In the case herein, the patient had classical cor triatriatum without a shunt or escape route; however, she had a long symptom-free period because of the large membrane communication. There are few reports regarding the relationship between the exact fenestration size and disease progress; however, by reviewing the literature, a 1.5$\mathrm{cm}$ diameter membrane fenestration appears to be large and classified in group III of Loeffler's classification [7].

Although TTE alone can diagnose most cases of cor triatriatum, TEE has the advantage of better visualizing the LA, the LA appendage, the membrane, and the pulmonary veins, hence providing higher sensitivity [8]. Cardiac CT and MRI provide better spatial resolution and superior tissue contrast compared with echocardiography. Moreover, cardiac CT and MRI can be performed easily in multiple planes [4]. Intra-operative TEE was not used in this case because the pre-operative cardiac evaluation showed only mild MS physiology with a large membrane fenestration, mild MR, and mild pulmonary hypertension, which was in the near-normal range. Intraoperative TEE is recommended to those who are more affected as it provides information about real-time hemodynamic status.

Patients with obstructive symptoms at any age should undergo surgical correction or percutaneous balloon dilatation of the fenestrations [1]. Most younger patients need surgical intervention because they are more likely to have significant degrees of trans-membrane flow obstruction [9]. However, adult onset cor triatriatum with minimal symptoms may not require surgical intervention, with conservative treatment sufficing. Percutaneous balloon dilatation is less invasive than surgery; however, subtle techniques and experience are required and long-term outcomes remain to be determined [10]. In the case herein, close monitoring was required as related symptoms progressed, and surgery appeared to be needed at the critical point.

As cor triatriatum presents with MS physiology, the main
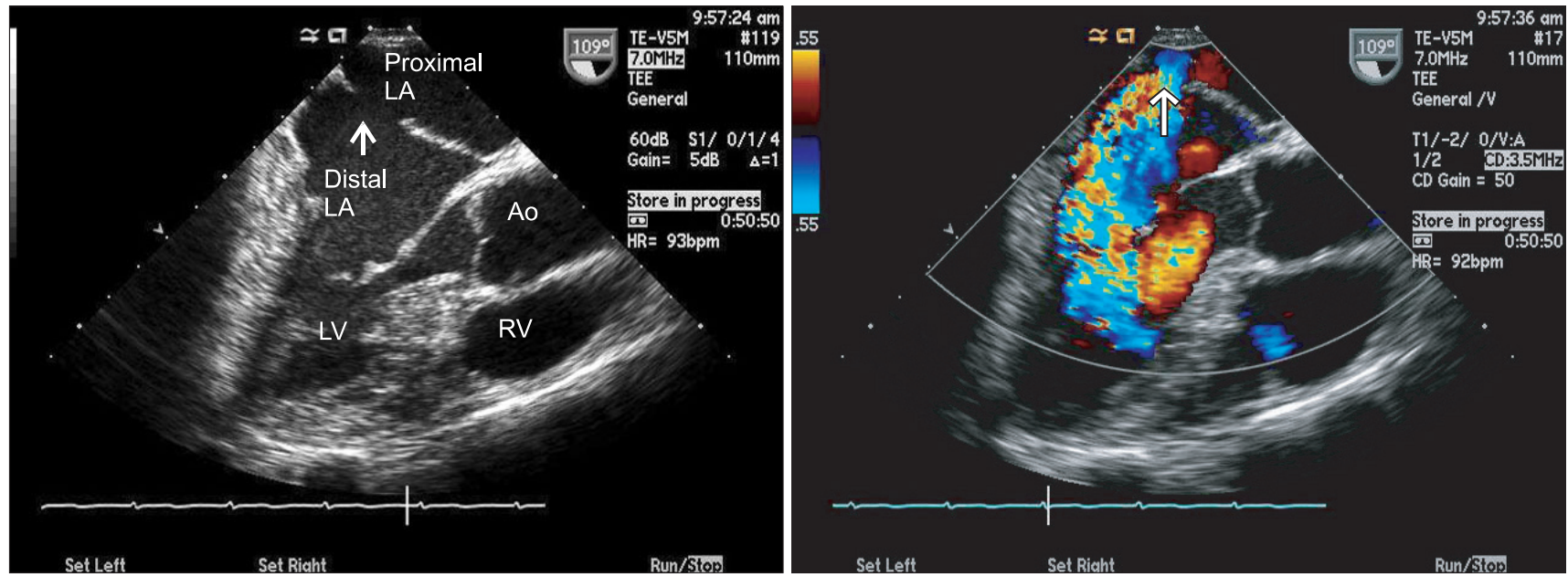

Fig. 3. (A) Trans-esophageal echocardiogram in the long-axis view. (B) Trans-esophageal echocardiogram of color-flow image in the same view. A membranous structure is dividing the LA (arrow) and blood flows through the membrane fenestration during ventricular diastole. 
anesthetic goals are maintaining adequate left ventricular diastolic filling and optimizing right heart function [3]. Therefore, tachycardia, pulmonary hypertension, and right heart failure (RHF) should be avoided and sinus rhythm, good cardiac contractility, adequate intraventricular volume (preload), and systemic vascular resistance (SVR; afterload) should be maintained. As tachycardia increases the LA and pulmonary pressures, hypovolemia and vasodilation should be avoided and treated promptly with fluids and pure alpha agonists, especially in the induction period. However, excessive fluid administration in the setting of existing increased LA pressure can lead to pulmonary edema and RHF, thus tight control of vascular tone and intravascular volume are necessary. Beta adrenergic antagonists can be used to lower the HR and dobutamine or milrinone may be ideal to use in cases of RHF with pulmonary hypertension because it has both inotropic and pulmonary vasodilatory effects. All anesthetics should be titrated carefully to avoid excessive vasodilation and cardiac depression, therefore concomitant use of opioids are beneficial to a volatile agent alone. Etomidate was used as an induction agent in this case because of its very stable hemodynamic profile; etomidate does not induce tachycardia or hypotension, and maintains the SVR. However, up to $80 \%$ of patients who were not pre-medicated with opioids experience myoclonus [11]. Remifentanil is known to reduce myoclonus induced by etomidate injection [12], and attenuates the increase in HR during intubation and surgical stress. Remifentanil also has a minimally depressive action on the cardiovascular system and provides excellent analgesia, thus avoiding tachycardia and hypertension. Myoclonus was not observed in this case and the HR and BP were maintained within acceptable ranges due to the remifentanil infusion.

In patients showing MS physiology, central neuraxial blockade could be dangerous because of the development of detrimental tachycardia. A sudden decrease in SVR may follow, therefore severe hemodynamic compromise could occur. However, a successful case of spinal anesthesia in cor triatriatum was reported [13]. If the obstruction is not severe, it can be a good alternative to general anesthesia, especially in patients with pulmonary edema or pulmonary hypertension. Epidural anesthesia seems to be more feasible than spinal anesthesia because hemodynamic change occurs more slowly. However, when anticoagulants are used due to AF, general anesthesia is preferred. In general anesthesia, pulmonary arterial pressure increases during the laryngoscopy and tracheal intubation, and adverse effects of positive-pressure ventilation on venous return could occur, ultimately leading to cardiac failure [14]. Moreover, $\mathrm{N}_{2} \mathrm{O}$ inhibits norepinephrine (NE) uptake by the lung and subsequent increases in plasma NE levels increase pulmonary vascular resistance (PVR) and pulmonary artery pressure [15]. $\mathrm{N}_{2} \mathrm{O}$ inhibition of NE uptake by the lung may be more pronounced in patients with pulmonary hypertension with increased intrathoracic pressure due to the pneumoperitoneum. A pneumoperitoneum also causes an increase in SVR, which may increase MR, and this probably contributes to a decrease in cardiac output [9]. However, in our patient, general anesthesia was conducted with an intraabdominal pressure maintained at $<10 \mathrm{mmHg}$ by our request. $\mathrm{N}_{2} \mathrm{O}$ was used with the trendelenberg position; however, the PIP was maintained at $<20 \mathrm{mmHg}$ by reducing the $\mathrm{V}_{\mathrm{T}}$ and increasing the RR. A HR at $65-90$ beats/min was thought to be optimal because the patient presented herein had mixed lesions of MS and MR physiology, which offered both proper diastolic filling time and decreased regurgitation. Although invasive monitors were not used in the current case, PCWP, CVP, and direct arterial monitoring devices are needed for major surgery with large fluid shifts or patients who are severely affected.

In summary, general anesthesia was administered to a patient with cor triatriatum uneventfully without invasive monitors. However, careful hemodynamic management with invasive monitors and intra-operative TEE are recommended for those patients who are severely affected. Successful peri-operative management should include prevention of tachycardia and pulmonary hypertension.

\section{References}

1. Alphonso N, Nørgaard MA, Newcomb A, d'Udekem Y, Brizard CP, Cochrane A. Cor triatriatum: presentation, diagnosis and long-term surgical results. Ann Thorac Surg 2005; 80: 1666-71.

2. Chen Q, Guhathakurta S, Vadalapali G, Nalladaru Z, Easthope RN, Sharma AK. Cor triatriatum in adults: three new cases and a brief review. Tex Heart Inst J 1999; 26: 206-10.

3. Frogel J, Galusca D. Anesthetic considerations for patients with advanced valvular heart disease undergoing noncardiac surgery. Anesthesiol Clin 2010; 28: 67-85.

4. Joe BN, Poustchi-Amin M, Woodard PK. Case 56: cor triatriatum dexter. Radiology 2003; 226: 701-5.

5. LeClair SJ, Funk KJ, Goff DR. Cor triatriatum presenting as postcesarean section pulmonary edema. J Cardiothorac Vasc Anesth 1996; 10: 638-9.

6. Lin YJ, Lee PC, Meng CC, Hwang B. Cor triatriatum with repeated episodes of syncope in an eighteen month-old girl: a rare cause of cardiogenic syncope. Int Heart J 2005; 46: 915-22.

7. LOEFFLER E. Unusual malformation of the left atrium; pulmonary sinus. Arch Pathol (Chic) 1949; 48: 371-6.

8. Einav E, Perk G, Kronzon I. Three-dimensional transthoracic echocardiographic evaluation of cor triatriatum. Eur J Echocardiogr 2008; 9: 110-2.

9. Slight RD, Nzewi OC, Buell R, Mankad PS. Cor-triatriatum sinister presenting in the adult as mitral stenosis: an analysis of factors which may be relevant in late presentation. Heart Lung Circ 2005; 14: 8-12. 
10. Kerkar P, Vora A, Kulkarni H, Narula D, Goyal V, Dalvi B. Percutaneous balloon dilatation of cor triatriatum sinister. Am Heart J 1996; 132: 888-91.

11. Kelsaka E, Karakaya D, Sarihasan B, Baris S. Remifentanil pretreatment reduces myoclonus after etomidate. J Clin Anesth 2006; 18: 83-6.

12. Lee SW, Gill HJ, Park SC, Kim JY, Kim JH, Lee JY, et al. The effect of remifentanil for reducing myoclonus during induction of anesthesia with etomidate. Korean J Anesthesiol 2009; 57: 438-43.
13. Kanbara T, Miyake T, Murao K, Jomura S, Yamada M, Sato N, et al. Anesthesia for cesarean section in a patient with cor triatriatum. Masui 2005; 54: 160-2.

14. Coskun D, Mahli A, Korkmaz S, Demir FS, Inan GK, Erer D, et al. Anaesthesia for caesarean section in the presence of multivalvular heart disease and severe pulmonary hypertension: a case report. Cases J 2009; 2: 9383.

15. Miller RD, Eriksson LI, Fleisher LA, Wiener-Kronish JP, Young WL. Miller's anesthesia. 7th ed. Philadelphia, Elsvier Inc. 2010, p 625. 